\title{
Autoimmune Hemorrhaphilia Resulting from Autoantibody against the A Subunit of Factor XIII
}

\author{
Emi Uchida ${ }^{1}$, Ken Watanabe ${ }^{1}$, Reina Arai $^{2}$, Masahide Yamamoto ${ }^{1}$, Masayoshi Souri ${ }^{3}$, \\ Tsukasa Osaki ${ }^{3}$, Akitada Ichinose ${ }^{3}$, Osamu Miura ${ }^{1}$ and Takatoshi Koyama ${ }^{1,2}$
}

\begin{abstract}
A 65-year-old woman was admitted with acute intramuscular hemorrhage of the left gluteus medius and piriformis muscles and associated anemia. Blood tests showed low plasma factor XIII (FXIII) antigen and activity. A cross-mixing test revealed a concave "inhibitor" pattern and anti-FXIII-A subunit antibody was detected. The patient was diagnosed with autoimmune hemorrhaphilia resulting from anti-FXIII antibody. The bleeding has not recurred since the initiation of treatment with oral immunosuppressive agents. Although hemorrhagic acquired FXIII deficiency is a rare disorder, prompt recognition of the underlying mechanism can save lives.
\end{abstract}

Key words: autoimmune hemorrhaphilia, anti-FXIII antibody, A subunit

(Intern Med 54: 2383-2387, 2015)

(DOI: 10.2169/internalmedicine.54.4791)

\section{Introduction}

Factor XIII (FXIII) is a coagulation factor whose activated form contributes to hemostasis by promoting crosslinks between fibrin monomers and stabilizing fibrin clots. FXIII is a pro-enzyme of two enzymatic A subunits (FXIIIA) and two non-catalytic B subunits (FXIII-B). Acquired factor XIII deficiency is a relatively common disorder, caused by decreased synthesis or increased consumption of FXIII (1). Various conditions have been associated with acquired FXIII deficiency, including disseminated intravascular coagulation, major surgery, liver diseases, sepsis, HenochSchönlein purpura, and inflammatory bowel disorders (such as Crohn's disease and ulcerative colitis). The FXIII deficiency is mild and critical bleeding is rare. Conversely, autoimmune hemorrhaphilia resulting from anti-FXIII antibody (AHXIII) is a very rare, life-threatening bleeding disorder observed mostly in elderly patients. Only a total of 83 AHXIII cases have so far been diagnosed worldwide (2). AHXIII tends to become chronic and intractable. This disorder is not always recognized, and a late diagnosis may lead to patient death due to severe bleeding. We herein report a case of AHXIII, where we promptly diagnosed, properly treated, and characterized the inhibitor in detail.

\section{Case Report}

A 65-year-old woman was admitted to our hospital with pain and a broad subcutaneous hemorrhage over the left thigh and buttocks. She had a history of acquired hypothyroidism due to Hashimoto's chronic thyroiditis since 20 years of age, type 2 diabetes, and chronic hepatitis $\mathrm{C}$ since 41 years of age; however, she had no prior history of bleeding tendency. The patient had received thyroid hormone replacement therapy. None of her family members had a history of bleeding. The patient had experienced pain in her right thigh when carrying heavy laundry up and down stairs, and pain began in her left thigh and buttocks 1 week later. On presentation at our hospital, the patient had a broad subcutaneous hemorrhage on the left thigh. Computed tomography revealed an intramuscular hemorrhage of the left gluteus medius and piriformis muscles (Fig. 1). Her hemoglobin level was $8.1 \mathrm{~g} / \mathrm{dL}$, which was down from $13.4 \mathrm{~g} / \mathrm{dL} 2$ months earlier. The patient was admitted to the Department of Vascular Surgery at our hospital and underwent em-

${ }^{1}$ Department of Hematology, Tokyo Medical and Dental University, Japan, ${ }^{2}$ Graduate School of Health Care Sciences, Tokyo Medical and Dental University, Japan and ${ }^{3}$ Department of Molecular Patho-Biochemistry and Patho-Biology, Yamagata University School of Medicine, Japan Received for publication December 22, 2014; Accepted for publication January 15, 2015

Correspondence to Dr. Takatoshi Koyama, koyama.lmg@tmd.ac.jp 

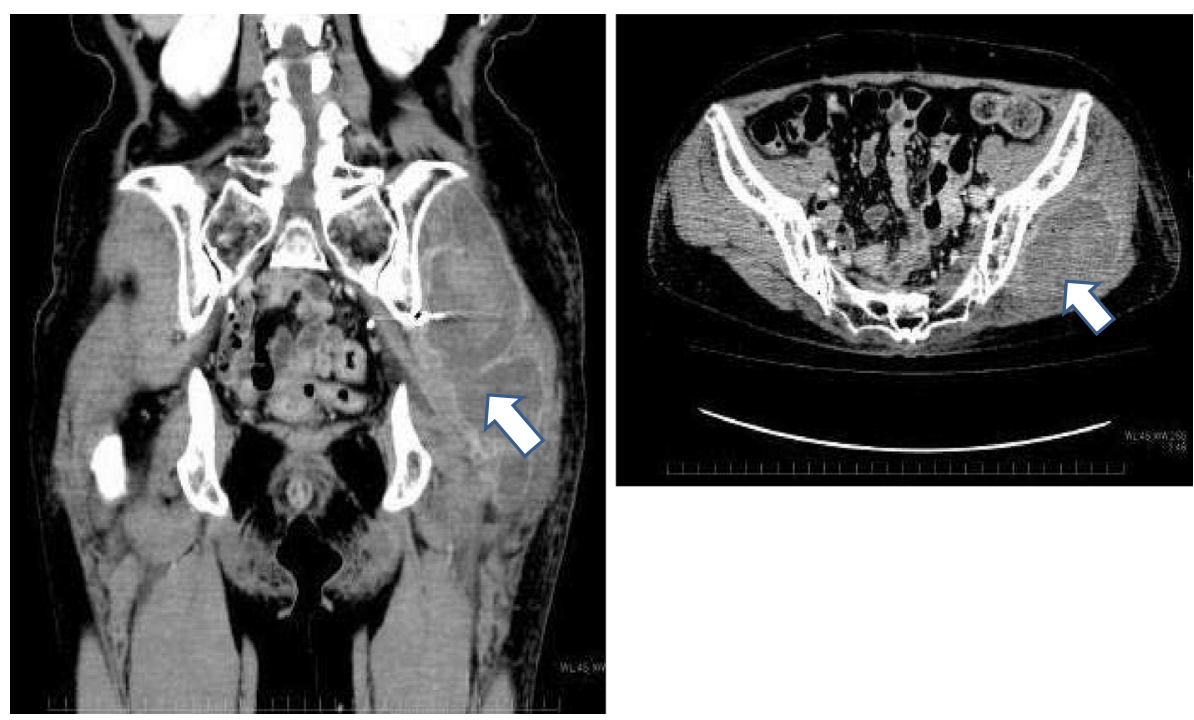

Figure 1. Intramuscular hemorrhage of the left gluteus medius and piriformis muscles confirmed by computed tomography on admission.

Table. Laboratory Data on Admission. Hemostatic Parameters Included Reduced Plasma Levels of FXIII Antigen and Activity.

\begin{tabular}{|c|c|c|c|c|}
\hline WBC & $5,800 / \mu \mathrm{L}$ & TP & $6.2 \mathrm{~g} / \mathrm{dL}$ & \multirow[t]{2}{*}{ bleeding time $4 \mathrm{~min}$} \\
\hline $\mathrm{Neu}$ & $60.5 \%$ & Alb & $3.4 \mathrm{~g} / \mathrm{dL}$ & \\
\hline Lym & $25.2 \%$ & BUN & $8 \mathrm{mg} / \mathrm{dL}$ & PT $\quad 10.5 \mathrm{sec}(10.5)$ \\
\hline Mo & $8.3 \%$ & $\mathrm{Cr}$ & $0.44 \mathrm{mg} / \mathrm{dL}$ & APTT $\quad 25.9 \mathrm{sec}(29.0)$ \\
\hline Eo & $5.5 \%$ & UA & $2.8 \mathrm{mg} / \mathrm{dL}$ & \multirow{2}{*}{$\begin{array}{lr}\text { FDP } & 5.4 \mu \mathrm{g} / \mathrm{mL} \\
\text { D-dimer } & 0.63 \mu \mathrm{g} / \mathrm{mL}\end{array}$} \\
\hline $\mathrm{Ba}$ & $0.5 \%$ & AST & $47 \mathrm{U} / \mathrm{L}$ & \\
\hline $\mathrm{RBC}$ & $254 \times 10^{4} / \mu \mathrm{L}$ & ALT & $46 \mathrm{U} / \mathrm{L}$ & \multirow{3}{*}{$\begin{array}{l}\text { total PAI-1 } 47 \mathrm{ng} / \mathrm{mL} \\
\text { plasmin inhibitor } 120 \%\end{array}$} \\
\hline $\mathrm{Hb}$ & $8.0 \mathrm{~g} / \mathrm{dL}$ & T-Bil & $1.6 \mathrm{mg} / \mathrm{dL}$ & \\
\hline $\mathrm{Ht}$ & $23.6 \%$ & CK & $771 \mathrm{U} / \mathrm{L}$ & \\
\hline Plt & $21.7 \times 10^{4} / \mu \mathrm{L}$ & CRP & $2.31 \mathrm{mg} / \mathrm{dL}$ & \multirow{3}{*}{$\begin{array}{lr}\text { FXIII Ag } & 3 \% \\
\text { FXIII activity } & 8 \% \\
\text { VWF activity } & 199 \%\end{array}$} \\
\hline \multicolumn{2}{|c|}{$<2$ months ago $>$} & \multicolumn{2}{|c|}{ HBs-Ag (-) } & \\
\hline WBC & $5,400 / \mu \mathrm{L}$ & HBs- $-A$ & & \\
\hline $\mathrm{RBC}$ & $431 \times 10^{4} / \mu \mathrm{L}$ & $\mathrm{HCV}-$ & $(+)$ & \\
\hline $\begin{array}{l}\mathrm{Hb} \\
\mathrm{Ht}\end{array}$ & $\begin{array}{l}13.4 \mathrm{~g} / \mathrm{dL} \\
38.3 \%\end{array}$ & & $(-)$ & \\
\hline Plt & $12.2 \times 10^{4} / \mu \mathrm{L}$ & & & \\
\hline
\end{tabular}

bolotherapy of the superior and inferior gluteal arteries. The Department of Vascular Surgery consulted us regarding this hemorrhage. Her routine hemostatic tests were normal, but we found that she had a low plasma FXIII antigen concentration of $3 \%$ and FXIII activity of $8 \%$ (Table). FXIII antigen was determined with a latex agglutination assay (NS auto-FXIII; Kainos, Tokyo, Japan). FXIII activity was determined with a photometric ammonia release assay (Berichrom FXIII; Dade Behring, Marburg, Germany). No other abnormal hemostatic laboratory data were documented associated with the hemorrhage. A cross-mixing test showed a concave "inhibitor" pattern and markedly low plasma FXIII-A antigen levels. In contrast, the FXIII-B antigen levels were only $50 \%$ of the normal range (Fig. 2A, B). AntiFXIII-A antibody was highly positive on an immuno-blot assay (Fig. 2C). This neutralizing anti-FXIII-A antibody should also induce the rapid clearance of FXIII-A and FXIII-B. A fibrin cross-linking study showed the lack of both $\gamma$-dimerization and $\alpha$-polymerization, indicating that fibrin-stabilization was markedly impaired (Fig. 2D). We diagnosed the patient with acquired FXIII deficiency with FXIII-A inhibitor, AHXIII $(1,2)$.

Because active hemorrhaging was not observed after embolotherapy, we did not administer FXIII and instead initiated oral prednisolone (PSL) therapy at $1 \mathrm{mg} / \mathrm{kg}(50 \mathrm{mg} /$ day) (Fig. 3). Because a substantial hemorrhage did not recur, except for mild subcutaneous purpura on the knee, we decreased PSL by $5 \mathrm{mg}$ every 2 weeks after 1 month of treatment. On day 73, the PSL dose was $30 \mathrm{mg} / \mathrm{day}$. The plasma FXIII activity and antigen levels mildly improved to approximately $12-22 \%$ and $20-23 \%$, respectively, after PSL was initiated, indicating that the inhibitor activity still remained. To treat this ongoing problem, we added cyclophosphamide (CPA) at $50 \mathrm{mg} /$ day to PSL (30 mg/day) beginning on day 94. Since that time, no hemorrhage has been documented, and the residual FXIII activity in the 1:1 crossmixing test increased from $7 \%$ to $40 \%$. Due to the patient's worsening diabetes and osteoporotic vertebral compression fractures, and because of PSL's insufficient inhibitoreradication effect, we gradually decreased the PSL dosage and continued CPA at $50 \mathrm{mg} /$ day. Even after the addition of CPA, the plasma FXIII activity and antigen levels did not increase to more than $26 \%$ and $22 \%$, respectively (Fig. 3). Because health insurance restrictions prevented us from administering anti-CD20 rituximab, which is not approved for AHXIII in Japan or at our university hospital, we replaced CPA with cyclosporin A (CyA) at $4 \mathrm{mg} / \mathrm{kg}$ (200 mg/day) along with $7 \mathrm{mg} /$ day of PSL beginning on day 204. The FXIII activity and antigen level remained low with the addition of CyA, but the residual FXIII activity increased (Fig. 3). In fact, total anti-FXIII-A IgG decreased markedly to less than $10 \%$ of the initial level at onset (data not shown). Furthermore, bleeding has not recurred. We gradually decreased the CyA dose and the patient is currently being maintained on $100 \mathrm{mg} /$ day of CyA and $5 \mathrm{mg} /$ day of PSL. 
(A) Amine-incorporation assay 5-Step mixing test

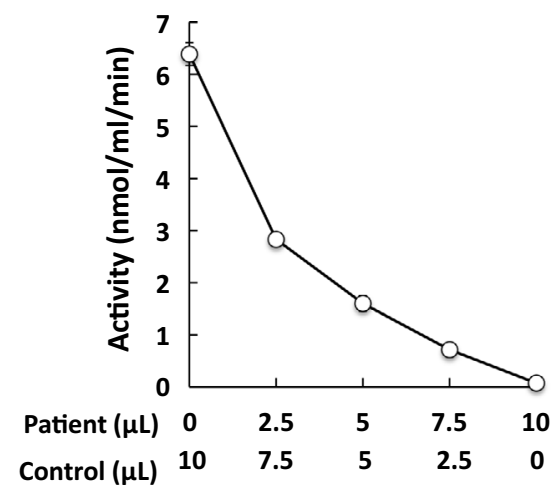

(B)

ELISA FXIII antigen in the patient's plasma

$\begin{array}{llclc} & \text { FXIII-A } & \text { FXIII-B } & \begin{array}{l}\text { A-bound FXIII-B } \\ \mathrm{U} / \mathrm{mL}(\%)\end{array} & \begin{array}{l}\text { Amine incorporation } \\ \text { pmol } / \mathrm{min} / \mathrm{mL}(\%)\end{array} \\ & \mathrm{U} / \mathrm{mL}(\%) & \mathrm{U} / \mathrm{mL}(\%) & 1.46 \pm 0.07(100) & 6.39 \pm 0.22(100) \\ \text { Control } & 1.39 \pm 0.06(100) & 1.10 \pm 0.03(100) & 1.46 \pm 0.03(1)\end{array}$

(C) Dot blot analysis of FXIII-reactive immunoglobulins

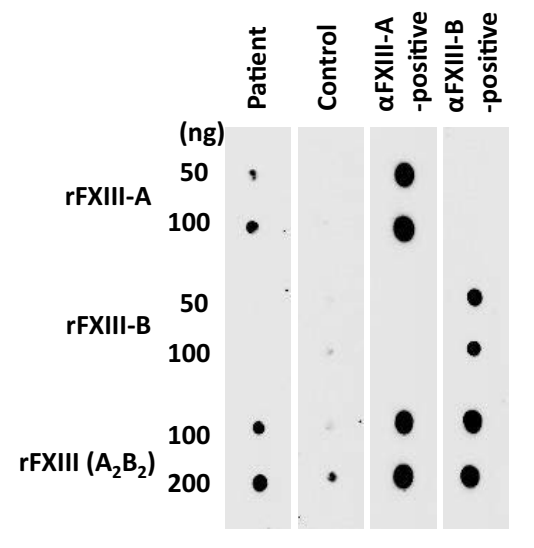

(D) Fibrin cross-linking

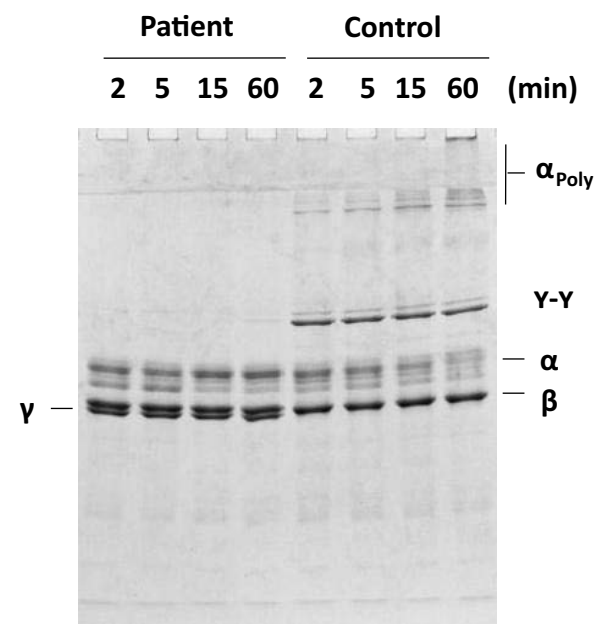

Figure 2. (A) Cross-mixing test. A five-step dilution cross-mixing test with an amine incorporation assay was performed using the patient's plasma at ratios of $0: 1,1: 3,1: 1,3: 1$, and 1: 0 with normal plasma. The mixed samples showed a concave "inhibitor" pattern. (B) Enzyme-linked immunosorbent assay (ELISA) of FXIII antigen in the patient's plasma. The plasma FXIII-A levels were markedly low. In contrast, the FXIII-B levels were reduced by no more than one-half. (C) Dot blot analysis of FXIII-reactive immunoglobulins. A dot blot analysis was performed using recombinant FXIII-A (rFXIII-A), recombinant FXIII-B (rFXIII-B), and their complexes [rFXIII $\left.\left(\mathrm{A}_{2} \mathrm{~B}_{2}\right)\right]$ at the indicated amounts, shown as ng of antigen. The positive controls were plasma from patients with previously confirmed AHXIII. The results showed that anti-FXIII-A antibody was positive, but anti-FXIII-B antibody was negative, indicated that the patient suffered from acquired factor XIII deficiency with FXIII-A inhibitor. (D) Fibrin cross-linking study. The fibrin cross-linking study was performed by adding $1 \mathrm{unit} / \mathrm{mL}$ thrombin and $5 \mathrm{mM} \mathrm{CaCl} 2$ to the patient's plasma and to normal control plasma. Clots were recovered at the indicated time intervals and subjected to sodium dodecyl sulfate-polyacrylamide gel electrophoresis. The results indicated the lack of both $\gamma$-dimerization and $\alpha$-polymerization. 


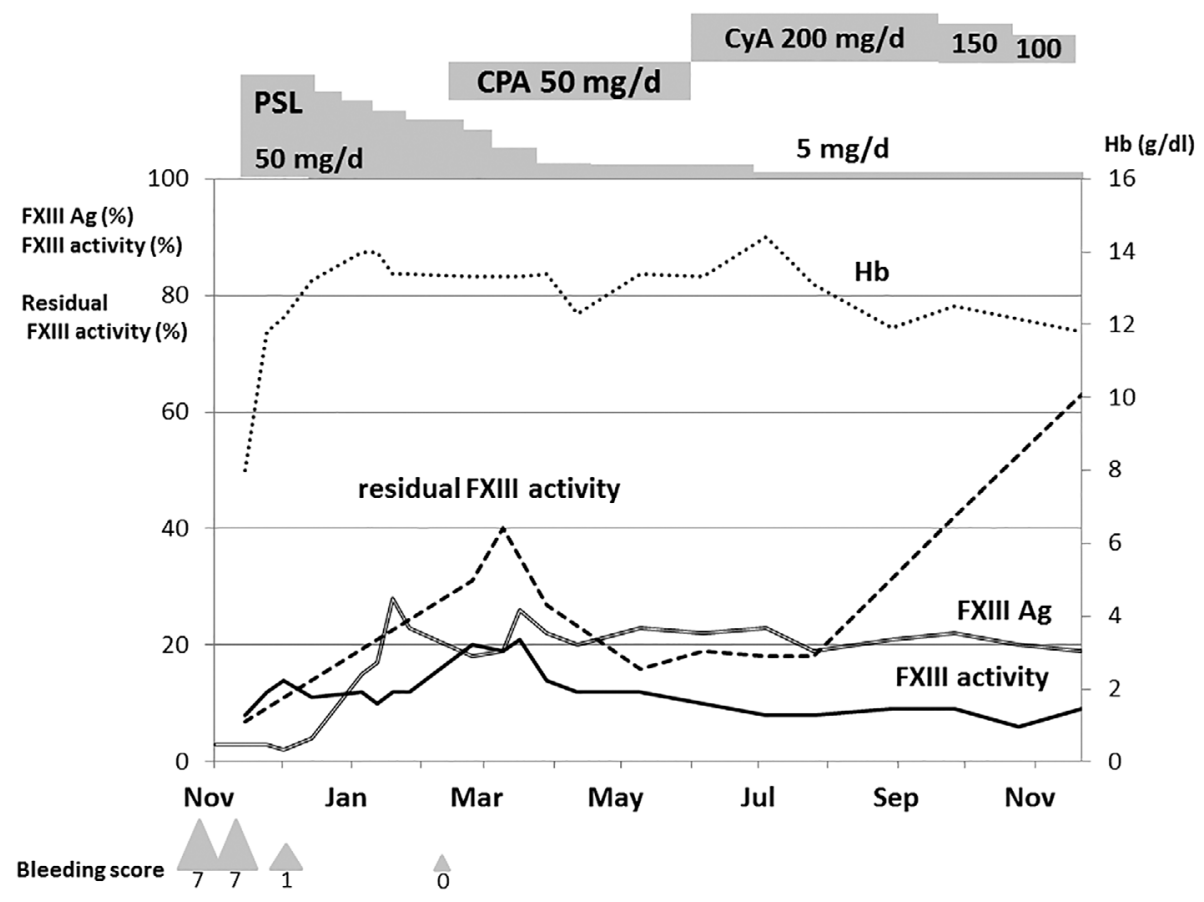

Figure 3. Clinical course after hospitalization. The plasma FXIII antigen levels were elevated mildly after starting prednisolone (PSL), but remained near 20\%. The FXIII activity levels were elevated only slightly at approximately $\mathbf{1 2 - 2 2 \%}$ with PSL, cyclophosphamide, or cyclosporine A as immunosuppressive treatments. The residual FXIII activity is the FXIII activity in a 1: 1 cross-mixing test incubated for 2 hours at $37^{\circ} \mathrm{C}$. A mild increase in the residual activity was observed with immunosuppressive treatment. A Japanese version of the ISTH/SSC Bleeding Assessment Tool was utilized to objectively evaluate the patient's bleeding symptoms (2). The bleeding score was 7 at admission to our hospital and promptly decreased following the administration of immunosuppressive medication.

\section{Discussion}

AHXIII occurs most commonly in the elderly, with a mean patient age of 70.4 years in Japan (2). Our patient was a 65-year-old woman and presented with typical spontaneous intramuscular bleeding. FXIII deficiency in children is generally congenital, but a case of a 9-year-old girl with AHXIII has been reported (3). Approximately half of all AHXIII cases are idiopathic and aging may be one of the most important factors for AHXIII, probably due to the loss of "self-immune tolerance" (2). The remaining half of the cases has some underlying disorders, such as autoimmune diseases and malignant tumors, or may be due to pharmaceutical agents, such as isoniazid, phenytoin, penicillin, and ciprofloxacin $(2,4,5)$. Severe liver disease may also cause FXIII deficiency because of decreased synthesis of FXIII-B in the liver; however, in the present case, chronic hepatitis $\mathrm{C}$ was well controlled and the patient had an inhibitor against FXIII-A. The present patient also had diabetes mellitus and autoimmune hypothyroidism, both of which were well controlled. The relationship between acquired von Willebrand syndrome and hypothyroidism is well documented (6), and AHXIII has been reported in a patient with congenital hypothyroidism (7), however, there are no current reports of
AHXIII in patients with acquired hypothyroidism. Our patient had a history of Hashimoto's chronic thyroiditis for more than 40 years, and the onset of AHXIII was not found to be associated with the deterioration of thyroid function. While the loss of "self-immune tolerance" may be common between Hashimoto's thyroiditis and AHXIII, a direct relationship between those disorders has not yet been shown.

FXIII concentrate, fresh frozen plasma, and cryoprecipitate have been used to control bleeding in patients with AHXIII. To eradicate coagulation inhibitors, corticosteroids, CPA, CyA, and rituximab are typically administered, according to the acquired hemophilia guidelines $(2,8)$. In the present case, we initially treated the patient with PSL, which was mildly effective. Neither CPA nor CyA were effective. The efficacy of rituximab for acquired inhibitors of coagulation factors, including AHXIII, has been reported in several studies $(9,10)$. However, we were not able to use rituximab in this case because its use for this disorder is not currently accepted at our hospital. In other previously studies in which steroids, CPA, and CyA were ineffective $(11,12)$, alternative treatments, such as plasmapheresis, immunoglobulin, and rituximab, were used, resulting in increased FXIII activity. In the present case, active bleeding has not recurred since the patient began PSL treatment, which represents clinical remission. Thus, a mild increase in the FXIII anti- 
gen and activity and residual FXIII activity may be sufficient for hemostasis. However, an increased risk of bleeding persists because the patient's FXIII activity remains low, which means that laboratory or immunological remission (2) has not yet been obtained. We must closely follow the patient for a long period, and if bleeding recurs, we plan to administer steroid pulse therapy with methyl-PSL with or without FXIII concentrate. Because FXIII is a rather polymorphic protein (13), and because a FXIII concentrate "Fibrogammin ${ }^{\circledR}$ " made from Caucasian plasma is now available in Japan, a booster effect of the FXIII concentrate on the production of anti-FXIII antibody is anticipated. Therefore, we have refrained from infusing the FXIII concentrate in the present case where the bleeding was not severe. The "watch and wait" strategy, along with the maintenance of mild immunosuppressive therapies, seems to be clinically relevant for older patients with risks of serious adverse events, such as in the present case.

Hemorrhagic cases of acquired inhibitors of coagulation factors, such as acquired hemophilia A, have been on the rise in Japan, which has become the leading "super-aging" society. In FXIII deficiency, the results of routine coagulation tests (such as prothrombin time and activated partial thromboplastin time) are normal. As a result, some patients with FXIII deficiency may be misdiagnosed. Moreover, AHXIII patients may bleed profusely. While intramuscular and subcutaneous bleeding are common, most fatal bleeding is intracranial $(2,5)$. In some patients, this disorder is diagnosed after autopsy in massive and fatal bleeding cases (14). To decrease the number of bleeding deaths among patients with AHXIII, we have to carefully examine hemostatic tests, including FXIII antigen and activity, to diagnose AHXIII and begin treatment as early as possible. The full characterization of the inhibitor as presented in this report may also be essential in initiating the most appropriate antihemorrhagic therapy and to remove related conditions to eradicate the inhibitor and to improve the patient's outcome (5). More nationwide and worldwide surveying is necessary to establish the treatment strategies.

\section{The authors state that they have no Conflict of Interest (COI).}

\section{Acknowledgement}

We greatly thank Dr. Soutaro Katsui (Department of Vascular Surgery, Tokyo Medical and Dental University) for the referral of this patient to our department.

This report was presented in part at the 604th Kanto regional meeting of the Japanese Society of Internal Medicine held in Tokyo, Japan where it won the Incentive Award

\section{Financial Support}

The analyses of FXIII inhibitor were supported by a research grant from the Japanese Ministry of Health, Labour and Welfare.

\section{References}

1. Ichinose A. Factor XIII is a key molecule at the intersection of coagulation and fibrinolysis as well as inflammation and infection control. Int J Hematol 95: 362-370, 2012.

2. Ichinose A. Inhibitors of Factor XIII/13 in older patients. Semin Thromb Hemost 40: 704-711, 2014.

3. Kessel R, Hu C, Shore-Lesserson L, Rand J, Manwani D. A child with acquired factor XIII deficiency: case report and literature review. Haemophilia 19: 814-826, 2013.

4. Ichinose A, Souri M; Japanese collaborative research group on "Acquired haemorrhaphilia due to factor XIII deficiency". As many as 12 cases with haemorrhagic acquired factor XIII deficiency due to its inhibitors were recently found in Japan. Thromb Haemost 105: 925-927, 2011.

5. Franchini M, Frattini F, Crestani S, Bonfanti C. Acquired FXIII inhibitors: a systematic review. J Thromb Thrombolysis 36: 109114, 2013.

6. Manfredi E, van Zaane B, Gerdes VE, Brandjes DP, Squizzato A. Hypothyroidism and acquired von Willebrand's syndrome: a systematic review. Haemophilia 14: 423-433, 2008.

7. Fear JD, Miloszewski KJ, Losowsky MS. An acquired inhibitor of factor XIII with a qualitative abnormality of fibrin cross-linking. Acta Haematol 71: 304-309, 1984.

8. Huth-Kühne A, Baudo F, Collins $\mathrm{P}$, et al. International recommendations on the diagnosis and treatment of patients with acquired hemophilia A. Haematologica 94: 566-575, 2009.

9. Franchini M, Veneri D, Lippi G, Stenner R. The efficacy of rituxi$\mathrm{mab}$ in the treatment of inhibitor-associated hemostatic disorders. Thromb Haemost 96: 119-125, 2006.

10. Ogawa Y, Mihara M, Souri M, et al. Complete remission achieved by steroid pulse therapy following rituximab treatment in a case with autoimmune haemorrhaphilia due to anti-factor XIII antibodies. Thromb Haemost 112: 831-833, 2014.

11. Miesbach W. Rituximab in the treatment of factor XIII inhibitor possibly caused by Ciprofloxacin. Thromb Haemost 93: 10011003, 2005.

12. Ajzner É, Schlammadinger Á, Kerényi A, et al. Severe bleeding complications caused by an autoantibody against the B subunit of plasma factor XIII: a novel form of acquired factor XIII deficiency. Blood 113: 723-725, 2009.

13. Okumura T, Yamada T, Park SC, Ichinose A. No Val34Leu polymorphism of the gene for factor XIIIA subunit was detected by ARMS-RACE method in three Asian populations. J Thromb Haemost 1: 1856-1857, 2003.

14. Sugiyama H, Uesugi H, Suzuki S, Tanaka K, Souri M, Ichinose A. Aggressive fatal case of autoimmune hemorrhaphilia resulting from anti-Factor XIII antibodies. Blood Coagul Fibrinolysis 24: 85-89, 2013.

(C) 2015 The Japanese Society of Internal Medicine

http://www.naika.or.jp/imonline/index.html 